\title{
DIGITAL TECHNOLOGY The mixed reality medical ward round with the MS HoloLens 2: Innovation in reducing COVID-19 transmission and PPE usage
}

\author{
Authors: Jeremy B Levy, ${ }^{A}$ Edmund Kong, ${ }^{B}$ Nathan Johnson, ${ }^{B}$ Ashni Khetarpal, ${ }^{B}$ James Tomlinson, ${ }^{A}$ Guy FK Martin ${ }^{C}$ \\ and Anisha Tanna ${ }^{\mathrm{A}}$
}

\section{Background}

The COVID-19 pandemic necessitated changes to the traditional medical ward round to protect staff and patients. This study investigated the value and acceptability of using the Microsoft HoloLens 2 mixed reality headset in a COVID-19 renal medicine ward.

\section{Methods}

The HoloLens 2 was used during the height of the COVID-19 pandemic and it was compared with the days prior to its introduction. Staff exposure to COVID-19 and PPE usage were measured, and staff and patients were surveyed on the HoloLens 2 experience.

Results

The average ward round was significantly shorter with the use of the HoloLens 2 ( 94 minutes vs 137 minutes; $p=0.006$ ). With the HoloLens 2 , only the consultant was in direct contact with COVID-19 patients compared with up to seven staff members on a normal ward round. Personal protective equipment usage was reduced by over $50 \%$. Both staff and patients were positive about its use but raised some important concerns.

\section{Conclusion}

The HoloLens 2 mixed reality technology is an innovative solution to the challenges posed by COVID-19 to the traditional medical ward round.

KEYWORDS: COVID-19, mixed reality technology, Microsoft HoloLens 2, PPE, augmented reality

DOI: $10.7861 /$ fhj.2020-0146

Authors: A consultant nephrologist, Imperial College Healthcare NHS Trust, London, UK; ${ }^{B}$ foundation trainee, Imperial College Healthcare NHS Trust, London, UK; ' ${ }^{c}$ linical lecturer in surgery, Imperial College Healthcare NHS Trust, London, UK

\section{Introduction}

The COVID-19 pandemic has presented numerous challenges to the traditional hospital ward round. The need to minimise staff exposure to patients infected with SARS-CoV-2 has resulted in only the most senior clinicians entering patient areas on ward rounds. This increases the risk of communication errors as the senior clinician can only relay information to the rest of the team upon leaving the patient area. Nor are clinicians able to view clinical results and observations while seeing patients, as taking these records into COVID-19 areas would be an infection control risk. There are also continuity of care issues as the majority of the team cannot physically see the patients on a ward round and will therefore be less familiar with them at later times.

Innovative solutions are needed to solve these issues and maintain high quality patient care. Some teams use mobile phones and encrypted applications such as WhatsApp, leaving a channel of communication open when one clinician is seeing the patient, so that the distanced team can hear the conversation or answer questions. New technologies such as mixed reality headsets are one potential solution. These devices use cameras and microphones allowing two-way communication, and simultaneously utilise augmented reality by allowing the wearer to map digital images onto the physical world which the user can interact (eg using hand gestures).

The Microsoft (MS) HoloLens 2 is a mixed reality headset. This device incorporates a holographic heads-up display (HUD) on the visor, onto which patient results, observations and other information can be presented. The inbuilt camera and microphone allow for bidirectional audio and visual communication between the wearer and (multiple) remote users. The HoloLens 2 can be worn by the clinician as they interact with patients, while the remainder of the healthcare team see a first person view from the HoloLens 2 wearer on a computer, allowing two-way communication with the clinician from a remote location. The HoloLens 2 has the potential to solve some of the challenges COVID-19 presents to the traditional ward round such as reducing personal protective equipment (PPE) usage, as it allows the medical team to engage in ward rounds remotely from a clean area.

There are many studies on the use of augmented reality for surgery in the operating room, in the emergency department and for medical education. ${ }^{2-4}$ Cafferkey et al have also recently 
reported the use of HoloLens 2 to reduce exposure of healthcare professionals (HCPs) to aerosol generating procedures (AGPs). ${ }^{5}$ However, there are no studies on its usefulness for medical ward rounds. This study was a pilot conducted at the height of the COVID-19 pandemic in London, and aimed to ascertain the value and acceptability of using the HoloLens 2 in a renal medicine ward that cared for patients with COVID-19.

\section{Methods}

Study design

Over a 2-week period, the HoloLens 2 was used during the service reconfigurations imposed by the COVID-19 pandemic to conduct the morning consultant ward round on a 21 -bed renal medicine ward full of patients infected with SARS-CoV-2 at Hammersmith Hospital, London. Patients were in four-bedded bays or in single occupancy side rooms. The consultant physician wore the HoloLens 2 headset (Fig 1) while seeing patients in person. The remainder of the multidisciplinary team (MDT), including junior doctors, nurses and pharmacists, remained in a separate room for the duration of the ward round. MS Teams was the communications software used to host the call between the HoloLens 2 device and the desktop computer(s) in the doctor's office. Video footage and audio of the consultant reviewing patients was live streamed to the remainder of the medical team using the HoloLens 2. Direct audio communication between the consultant and the medical team was possible via the HoloLens

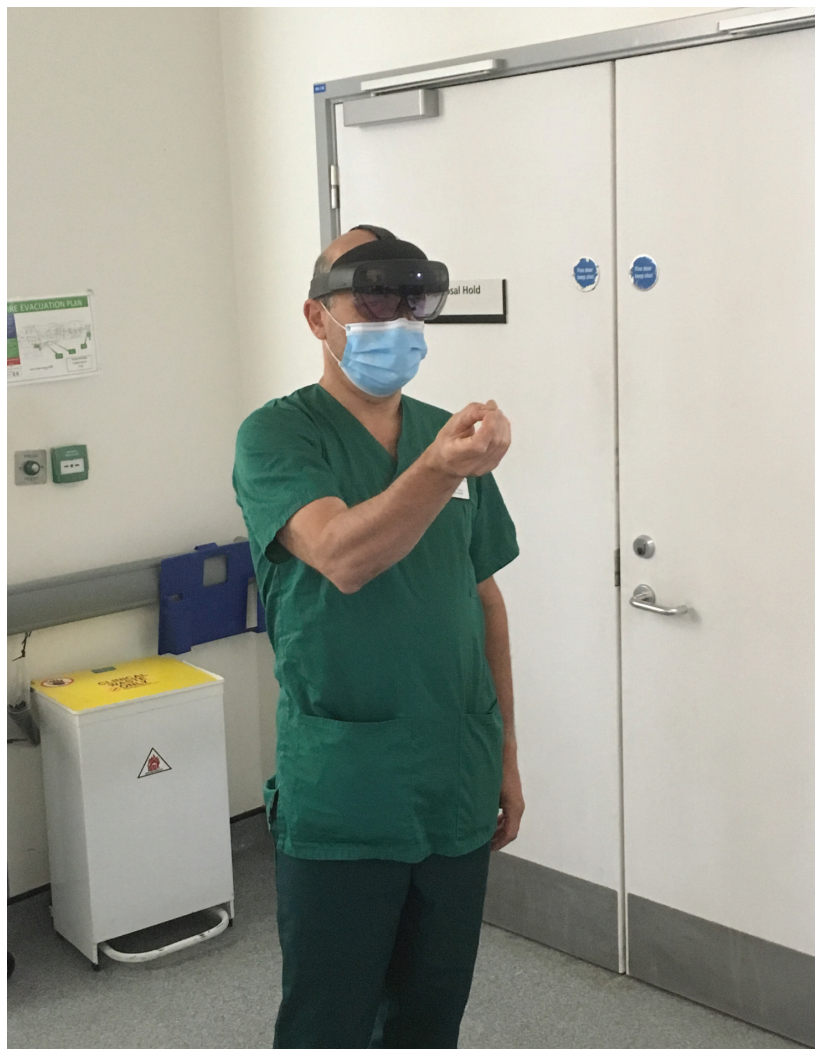

Fig 1. One of the authors wearing the HoloLens 2 and manipulating a holographic X-ray.
2; and the headset user was able to see applications being shared from the office computer onto the HUD including previous clinical note entries in the electronic record, clinical observations, pathology results and imaging. We collected qualitative feedback from patients and staff on the use of the HoloLens 2 in the ward setting during the COVID-19 pandemic, and quantitative data to estimate benefits on potential for exposure to infection.

\section{Qualitative data on staff and patient opinion of HoloLens 2}

An online survey platform (Qualtrics) was used to create an anonymous survey to gather the opinions of the MDT and patients on the use of HoloLens 2 for ward rounds. Data collection for patients' feedback was performed over 2 random days when the HoloLens 2 was used. Two of the authors conducted the patient survey by asking the designated questions to the patients in person. Patients selected were all those who were cognitively intact, able to communicate in English, and clinically stable at the time of the ward round and survey. Questions included feedback on various aspects of their experience being reviewed by a consultant using the HoloLens 2.

Quantitative data on number of HCP in contact with COVID-19 patients, duration of exposure and PPE used

Six ward rounds without HoloLens 2 were compared with six ward rounds with HoloLens 2 on different days on the same renal COVID-19 ward. There were at least two different consultants doing the ward round in each group on the different days. The duration of HCP exposure to COVID-19 was indirectly measured by time taken to undertake a full ward round with and without HoloLens 2. This data also gave a measure of efficiency as any time saved on ward rounds could be used for other essential ward jobs involved in patient management. On most days the ward was at full capacity with 21 patients.

COVID-19 PPE guidance for the trust at the time of this study mandated change of plastic aprons and surgical masks between each 'clinical area' (ie bay or side room). Gloves were changed between each patient if the patient was examined, otherwise same gloves were used within each 'clinical area'. There were four bays and five side rooms in the ward, making nine clinical areas in total. The amount of each of these items used was counted on all 12 ward rounds during the data collection period.

This study was approved as a service evaluation by the Imperial College Renal and Transplant Quality and Safety (Governance) committee. The technology was implemented at the height of the COVID-19 pandemic to determine if it would reduce healthcare staff to exposure to SARS-CoV-2.

\section{Results}

\section{Patients' views on the use of HoloLens 2}

The surveys yielded responses from nine patients and seven staff. The nine patients (five male; four female) had a mean age 62.6 \pm 15.3 years (range $25-84$ years). Three of the patients did not notice any difference between ward rounds with or without use of the HoloLens 2, and in fact had not noticed the device in use at all. The other six patients noticed the consultant wearing the headset and engaging with it. All patients felt there was no detrimental impact on their medical care, experience or interaction with the consultant. 
They were able to have the same conversation and ask questions to the medical team as in a traditional ward round. Two patients felt the HoloLens 2 provided better infection control as fewer doctors were entering the patients' bay or side room. When asked about the potential long-term use of the HoloLens 2 for future ward rounds, four patients said they 'neither like nor dislike' the idea, two patients 'liked [it] somewhat' and two patients 'liked [it] a great deal'.

\section{Healthcare professionals' feedback}

Staff responses were obtained from one nurse, two pharmacists, two senior house officers, one registrar and one consultant ( 6 female; 1 male). Five staff members rated their overall experience with the HoloLens 2 in use for ward rounds as 'Good', one as 'Average' and one felt it was 'Excellent' overall. When asked about the potential long-term use of the HoloLens 2, five staff members 'Somewhat agree', whereas two staff members 'Somewhat disagree' with the idea.

The feedback from the staff survey can be summarised into four main themes: overall impression of mixed reality technology, infection control, efficiency of ward rounds, and communication and patient interaction.

\section{Overall impression of mixed reality technology}

Three staff members had an overall positive impression of the HoloLens 2, describing it as 'very cool', 'amazing' and 'great innovative' technology. One pharmacist acknowledged that it was 'interesting to see through the eyes of the doctor' which was 'brilliant' from 'an educational point of view'. Other staff members preferred it compared with using WhatsApp phone calls, and that it 'allowed participation of multiple team members without all crowding next to the bedside', which can be 'overwhelming for patients' at times. However, one of the consultants described frustration with the 'pop-up menus' appearing when performing normal actions/gestures such as washing hands and when examining patients, which the augmented reality kit recognised as commands to undertake actions in the virtual space.

\section{Infection control}

Six members of the team felt that using the HoloLens 2 allowed avoidance of unnecessary staff exposure to COVID-19 and therefore was useful to prevent infection. They reported fewer number of people were in contact with COVID-19 patients and therefore less PPE was used. For the consultant physician that was exposed, this was also for a shorter duration due to the shorter ward round. There was also 'less crowding on ward rounds'.

However, one staff member identified that there was 'lack of social distancing for the MDT', as the rest of the team was 'crammed in a small room'.

\section{Efficiency}

Three staff members believed that using the HoloLens 2 resulted in more efficient ward rounds in COVID-19 wards compared with the traditional ward round or using other communication modalities. One reason for this was that it allowed for 'simultaneous completion' of ward jobs while the ward round continued.

\section{Communication and patient interaction}

One doctor preferred seeing patients physically in person on the ward round, which they felt had several advantages, such as "not properly seeing the patient for the first time if called to assess the patient when unwell', and 'discussions on sensitive topics' would be easier 'if patients have seen your face before'. Another response highlighted that, due to reduced face-to-face contact with the patient when using the HoloLens 2, the patient 'does not get to meet the rest of the team looking after their care'. Audio was an issue, as the team could not hear the patient's voice clearly or at all, as reported by four members of staff. Three staff members reported reduced and limited interaction between the consultant and the rest of the team. One person highlighted the difficulty in having a 'full MDT conversation with lots of muting and unmuting' of the computer microphone to reduce background noise in the office room. They felt it was 'less communicative than in-person ward rounds'.

\section{Healthcare professionals in contact with COVID-19 patients}

Without use of HoloLens 2, there were seven staff members on the ward round (consultant, registrar, senior house officer, two foundation doctors, charge nurse and pharmacist) compared with just one (the consultant) when the HoloLens 2 was used. Without HoloLens 2, at least two HCPs (consultant and registrar) would be in direct contact with patients, entering the bay and examining them (during this COVID-19 period). The remaining five staff members would be in ward corridors to aid documentation and MDT management of each patient, but outside the rooms to avoid direct exposure to SARS-CoV-2. With the HoloLens 2, while the consultant physically saw the patients, the rest of the MDT were in an office room to view and make remote contributions and documentation.

\section{Duration of ward round (and exposure to COVID-19 areas)}

The average duration of the six ward rounds without HoloLens 2 was $137 \pm 18$ minutes compared with $94 \pm 23$ minutes $(p=0.006)$ with the HoloLens 2. Thus, ward rounds were approximately $30 \%$ shorter and saved on average 43 minutes of a clinicians' time.

\section{PPE usage}

On a traditional ward round in a COVID-19 ward with two clinicians entering patient bays and five joining from outside, approximately 23 surgical masks, 18 aprons and 18 or fewer pairs of gloves are used. However, with the HoloLens 2 , there is over $50 \%$ reduction in usage of all PPE items, with nine surgical masks, nine aprons and nine pairs of gloves being used on average.

\section{Discussion}

This was clearly a very small pilot study undertaken at the height of the COVID-19 pandemic. The numbers of staff, patients and ward rounds documented was small, partly due to the evolution of the pandemic, but also the availability of the HoloLens 2 headset for piloting. The patient survey was not completed anonymously, which potentially introduced bias as patients may have altered their responses to answers they felt staff wanted. However, this method was deemed safer than providing an electronic device or paper survey which may be an infection control risk. Despite these limitations, this pilot study has demonstrated that the HoloLens 2 device can be successfully used on a medical ward to conduct ward 
rounds quicker, more efficiently, exposing fewer staff to infection risks, and allowing use of less PPE, with no significant detriment to patient engagement or patient care. This has clear major potential benefits during a pandemic. Most staff and patients both approved of the use of HoloLens 2 on COVID-19 wards and all adapted to the technology quickly.

The main benefit perceived by staff was improved infection control as a direct result of fewer staff being exposed to patients with COVID-19. A Royal College of Physicians survey found that approximately $30 \%$ of doctors were off sick at some point during the COVID-19 pandemic (majority due to COVID-19 illness or self-isolating due a symptomatic household member). ${ }^{6}$ With such a high number of staff absences at a time of peak demand for healthcare, it is vital to protect the workforce from any unnecessary exposure to high-risk environments, and innovative technology like the HoloLens 2 offers just that. Another benefit reported by staff was improved efficiency of the ward round, as indicated by the $30 \%$ shorter ward round duration when the HoloLens 2 was used. This allowed members of the MDT to contribute to a concise ward round while also giving them enough time in the remainder of the day to carry out their other clinical duties. Doctors, in particular, felt they could multitask during the ward round by carrying out high-priority jobs like requesting procedures or scans alongside the remote ward round.

The shorter ward round duration was also a significant factor in reducing length of exposure to COVID-19. As Bromage noted, 'successful infection = exposure to virus $x$ time'? ' Therefore, less time exposed to patients with COVID-19 and their clinical areas containing aerosols and droplets means less chance of healthcare professionals transmitting the virus at work. This risk is then further reduced by use of PPE. Cafferkey et al have recently shown that the use of the HoloLens 2 reduced PPE usage in a ward with extensive use of non-invasive ventilation (continuous positive airway pressure; aerosol generating). ${ }^{5}$ Our study shows that use of HoloLens 2 can also reduce PPE usage by at least $50 \%$ on a medical ward with no aerosol generating procedures. Given the concerns regarding PPE shortages that have arisen during the COVID-19 pandemic, this is an important benefit of using the HoloLens 2 for medical ward rounds. ${ }^{5}$ The reduction in PPE resulting from fewer staff members on the ward round also directly linked with shorter duration of ward rounds, as donning and doffing PPE is a time-consuming process.

Finally, the success of a new technology is ultimately defined by its acceptability to the end-user and utility in improving patient care. From our staff survey it is clear that the majority of staff approved of the HoloLens 2 for ward rounds in the long term. Prochaska et al have previously shown that hospital inpatients are comfortable with the perception of doctors using wearable computer headsets such as the Google Glass and the HoloLens $2 .{ }^{8}$ However, we believe we are the first study to evaluate patient approval of such a device after it has been used for inpatient ward rounds. Patients did not feel their care was compromised in any way by the use of the HoloLens 2 . There was overall approval for its long-term use, however, not all staff felt the benefits significant in the absence of a life-threatening infectious risk from patients to staff. The technology was not without potential concerns. Some healthcare professionals felt it detracted from true MDT discussions about each patient, and that patients lost the opportunity to meet other members of the team they might be seeing at a later time point. There were some difficulties with communication. Staff watching remotely reported that the headset impeded their ability to communicate with patients as they struggled to hear what patients were saying due to the background noise cancellation feature on the headset, and had to transmit any questions from them via the headset user. Some of this can be rectified by an external Bluetooth microphone nearer to the patient. Patient feedback on the contrary did not identify any communication difficulties with the medical team as they were able to communicate one-on-one with the consultant doing the ward round. Recognising any down sides allows training and education of users to prevent them being ongoing issues.

\section{Conclusion}

This study demonstrates a clear benefit to the use of the HoloLens 2 on medical wards with COVID-19 patients. Its use is highly effective at reducing staff exposure and PPE usage, while also being acceptable to both staff and patients. Further study can be considered to determine the educational value of using the HoloLens 2 for ward rounds to teach medical students clinical medicine remotely, and engaging other members of an MDT from remote locations. The HoloLens 2 is a viable solution to the problems COVID-19 has posed to the traditional ward round on medical wards and should be considered for more widespread use.

\section{Acknowledgements}

This work is supported by the National Institute for Health Research (NIHR) Biomedical Research Centre (BRC) based at Imperial College Healthcare NHS Trust. Thanks to Mr James Kinross for provision of the HoloLens 2 headset, support and advice, and the Imperial PanSurg team.

\section{Conflicts of interest}

Microsoft provided support with supply of the device but had no role in the design, analysis, write up or review of the manuscript.

\section{References}

1 Microsoft. Microsoft HoloLens 2. Microsoft, 2019. www.microsoft. com/en-us/hololens

2 Tepper OM, Rudy HL, Lefkowitz A et al. Mixed reality with hololens: Where virtual reality meets augmented reality in the operating room. Plast Reconstr Surg 2017;140:1066-70.

3 Munzer BW, Khan MM, Shipman B, Mahajan P. Augmented reality in emergency medicine: A scoping review. J Med Internet Res 2019;21:e12368.

4 Balian S, McGovern SK, Abella BS, Blewer AL, Leary M. Feasibility of an augmented reality cardiopulmonary resuscitation training system for health care providers. Heliyon 2019;5:e02205.

5 Cafferkey J], Hampson DOP, Ross C et al. Using HoloLens to reduce staff exposure to aerosol generating procedures during a global pandemic. medRxiv 2020.05.24.20107193.

6 Royal College of Physicians. COVID-19 and its impact on NHS workforce. RCP, 2020.

7 Bromage E. The risks - know them - avoid them. Erin Bromage, 2020. www.erinbromage.com/post/the-risks-know-them-avoid-them

8 Prochaska MT, Press VG, Meltzer DO, Arora VM. Patient perceptions of wearable face-mounted computing technology and the effect on the doctor-patient relationship. Appl Clin Inform 2016;7:946-53.

Address for correspondence: Prof Jeremy Levy, Imperial Renal and Transplant Unit, Imperial College Healthcare NHS Trust, Hammersmith Hospital, Du Cane Road, London W12 OHS, UK. Email: j.levy@imperial.ac.uk

Twitter: @jeremyblevy 